\title{
ÂNGULO DE GIRO E ESPAÇAMENTO ENTRE CARREADORES EM SISTEMAS AUTOPROPELIDOS DE IRRIGAÇÃ̃O COM O ASPERSOR PLONA-RL400 ${ }^{1}$
}

\author{
GIULIANI DO PRADO ${ }^{2}$, ALBERTO COLOMBO ${ }^{3}$, PRISCILA F. BIAGIONI ${ }^{4}$
}

\begin{abstract}
RESUMO: Valores determinados em laboratório, de vazão, raio de alcance e do perfil radial de aplicação de água do aspersor canhão PLONA-RL400, foram utilizados em simulações digitais da uniformidade de aplicação de água desse aspersor operando, na ausência de ventos, com diferentes ângulos de giro e espaçamentos entre carreadores, em sistemas autopropelidos de irrigação. Os valores simulados de uniformidade de aplicação de água foram apresentados em três grupos distintos, cada um dos quais representando condições operacionais (bocal e pressão) que determinam a ocorrência da mesma forma geométrica (I, II ou III) do perfil radial adimensional de aplicação de água do aspersor avaliado. Para os perfis do tipo I, II e III, observou-se que espaçamentos de carreadores menores que $50 \%$ do diâmetro molhado ou situados entre 80 e $90 \%$ do diâmetro molhado proporcionaram os maiores valores de uniformidade. Em todas as formas geométricas do perfil, os melhores valores de uniformidade de aplicação de água foram obtidos com ângulos de giro do aspersor entre 180 e $210^{\circ}$.
\end{abstract}

PALAVRAS-CHAVE: perfil radial, simulações digitais, uniformidade da irrigação.

\section{WETTED ANGLE AND TOWPATH SPACING FOR TRAVELING GUN SYSTEMS WITH THE PLONA-RL400 GUN SPRINKLER MODEL}

\begin{abstract}
Values of flow rate, radius of throw and radial precipitation profile obtained in laboratory with the PLONA-RL400 gun sprinkler are presented. These values were used on digital simulations of water application uniformity, under no wind conditions, provided by traveling gun machines operating with this sprinkler under different combinations of wetted angle and towpath spacing. Simulated uniformity values were presented arranged under three different clusters, each one corresponding to a different set of sprinkler operational conditions (nozzle versus service pressure) that results on the same geometrical shape (I, II or III) of PLONA-RL400 radial precipitation profile. For the three profile shapes, it was observed that towpath spacings shorter than $50 \%$ of the sprinkler wetted diameter and on the range between 80 and $90 \%$ of the sprinkler wetted diameter provide higher uniformity values. For all geometrical shapes, it was observed that higher uniformity values were achieved when the sprinkler wetted angle is set to a range between 180 and $210^{\circ}$.
\end{abstract}

KEYWORDS: radial profile, digital simulations, irrigation uniformity.

\section{INTRODUÇÃO}

A uniformidade de aplicação de água dos equipamentos autopropelidos de irrigação depende, principalmente, das condições de vento durante a irrigação, das características do aspersor utilizado (pressão de serviço, diâmetro dos bocais, ângulo do jato, etc.), da uniformidade da velocidade de deslocamento e da pressão de serviço do aspersor durante o seu deslocamento no carreador e, também, da seleção criteriosa do ângulo de giro e do espaçamento entre carreadores. ROCHA et al. (2005) salientam que há falta de critérios específicos de dimensionamento, de uso generalizado,

\footnotetext{
${ }^{1}$ Trabalho apresentado no XXXV Congresso Brasileiro de Engenharia Agrícola.

${ }^{2}$ Engo Agrônomo, Doutorando em Engenharia Agrícola, Departamento de Engenharia, Universidade Federal de Lavras, Lavras MG, Fone: (0XX35) 3821.9046, giulianip@bol.com.br.

${ }^{3}$ Eng $^{\mathrm{o}}$ Agrônomo, Professor Adjunto, Departamento de Engenharia, Universidade Federal de Lavras, Lavras - MG.

${ }^{4}$ Estudante de Engenharia Agrícola, Universidade Federal de Lavras, Lavras - MG.

Recebido pelo Conselho Editorial em: 19-5-2006
}

Aprovado pelo Conselho Editorial em: 2-4-2007 
capazes de proporcionar desempenho satisfatório e competitivo desses sistemas em diferentes condições operacionais.

O estudo pioneiro de BITTINGER \& LONGENBAUGH (1962) demonstrou que o formato geométrico do perfil radial de aplicação de água, do aspersor operando de forma estacionária, deve ser considerado no processo de seleção do espaçamento entre carreadores em sistemas autopropelidos de irrigação. Para o ângulo de giro de $360^{\circ}$, esses autores demonstraram que aspersores apresentando perfil de aplicação de água de formato elíptico (perfil $\mathrm{C}$ de Christiansen) atingem maior uniformidade com valores de espaçamento entre carreadores próximos de $70 \%$ do diâmetro molhado, enquanto aspersores com perfil de aplicação de água de formato triangular (perfil B de Christiansen) apresentam maior uniformidade de aplicação de água quando o espaçamento entre carreadores corresponde a 50\% do diâmetro de alcance.

Um formato triangular do perfil de aplicação de água é também considerado por ROLIM \& PEREIRA (2005) para simulação da distribuição espacial da água em sistemas autopropelidos de irrigação. Por outro lado, KELLER \& BLIESNER (1990), por considerarem que os canhões utilizados em sistemas autopropelidos de irrigação apresentam perfil de aplicação de água que tem formato quase uniforme, muito próximo do formato dos perfis D e E de Christiansen, recomendam, para condição de ventos fracos, que o espaçamento entre carreadores seja próximo de $80 \%$ do diâmetro molhado. TARJUELO (1999) e BERNARDO et al. (2005) também referenciam essa recomendação de $80 \%$ do diâmetro molhado.

No entanto, devido à dificuldade de determinar o formato geométrico assumido pelo perfil radial de aplicação de água dos grandes canhões utilizados em sistemas autopropelidos, há dúvida quanto à aplicabilidade dessas recomendações em situações de campo. PRADO (2004), utilizando perfis radiais de aplicação de água do aspersor canhão PLONA-RL300, em simulações digitais da uniformidade de aplicação de água de sistemas autopropelidos de irrigação, verificou que, em algumas condições operacionais do aspersor canhão, os maiores valores de uniformidade correspondiam a valores de espaçamento entre carreadores que superavam $85 \%$ do diâmetro molhado.

No que se refere à seleção do ângulo de giro do aspersor, ROCHA et al. (2005) comentam que um ângulo de giro com valor próximo de $270^{\circ}$ é recomendado para sistemas autopropelidos de irrigação. As simulações de PRADO (2004), com o aspersor canhão PLONA-RL300 operando em sistemas autopropelidos, indicam que ângulo de giro de $240^{\circ}$ proporciona melhor distribuição de água do que aquela obtida como ângulo de $270^{\circ}$.

KELLER \& BLIESNER (1990), baseados em simulações da distribuição de água de um aspersor com perfil radial de aplicação de água de formato uniforme, recomendam ângulo de giro próximo de $210^{\circ}$. TARJUELO (1999), baseado em resultados de ensaios realizados no CEMAGREF, na França, recomenda valores de ângulo de giro entre 200 e $220^{\circ}$.

Tendo em vista a necessidade de avaliar a aplicabilidade das diferentes recomendações de ângulo de giro e espaçamento entre carreadores encontradas na literatura e, também, a necessidade de fornecer aos projetistas e usuários de sistemas autopropelidos, que operam com o aspersor canhão PLONA-RL400, recomendações quanto à maneira mais adequada de utilizar esses equipamentos, desenvolveu-se este trabalho com os seguintes objetivos: i) apresentar valores, determinados em laboratório, de vazão, raio de alcance e perfil radial de distribuição de água do aspersor canhão PLONA-RL400, e ii) aplicar as características técnicas determinadas em laboratório do aspersor canhão PLONA-RL400 em simulações da distribuição de água de sistemas autopropelidos, de forma a fornecer, aos projetistas e usuários de sistemas autopropelidos, subsídios quanto à seleção das combinações de ângulo de giro e espaçamento entre carreadores que resultam em maior uniformidade de aplicação de água. 


\section{MATERIAL E MÉTODOS}

O aspersor PLONA, modelo RL400, é um canhão hidráulico de reversão lenta, com flange de acoplamento de 4", ângulo de lançamento de jato de água de $24^{\circ}$ e giro proporcionado pelo impacto de um dos seus dois braços oscilantes. Esse aspersor tem bocal principal do tipo cônico, de latão, disponível em seis diâmetros distintos (34; 36; 38; 40; 42 e 44 mm).

As determinações da vazão, do raio de alcance e do perfil radial de distribuição de água foram realizadas na bancada de ensaio de aspersores do Laboratório de Hidráulica da Universidade Federal de Lavras - UFLA, seguindo a metodologia descrita por PRADO \& COLOMBO (2005). Foram realizados 24 ensaios, de forma a determinar esses parâmetros com o aspersor operando em cada um dos seis diâmetros de bocal principal disponíveis e em quatro valores distintos de pressão de serviço $(392 ; 490 ; 588$ e $686 \mathrm{kPa})$.

Os perfis radiais de aplicação de água determinados em laboratório foram adimesionalizados, segundo a metodologia apresentada por SOLOMON \& BEZDEK (1980). Os 24 perfis foram utilizados para gerar 24 diferentes conjuntos compostos de 20 valores ordenados de intensidade adimensional de aplicação de água, correspondentes a 20 valores crescentes de distância adimensional ao aspersor, que se estendem desde 2,5 até $97,5 \%$, em intervalos regulares de $5 \%$ do raio de alcance observado.

Com o objetivo de identificar os formatos geométricos típicos que podem ser utilizados para racionalizar a representação do perfil radial adimensional do aspersor canhão PLONA-RL400 em diferentes condições operacionais, esses 24 conjuntos foram submetidos à análise de agrupamento, executada com o uso do algoritmo"K-Means" de TOU \& GONZALES (1974).

Para a execução das simulações digitais que possibilitam identificar as melhores combinações de espaçamento entre carreadores e ângulo de giro do aspersor PLONA-RL400, foi desenvolvido um aplicativo computacional, que se utiliza do Visual Basic da planilha eletrônica do Excel. Conforme ilustrado no fluxograma apresentado na Figura 1, esse aplicativo estabelece, em função da combinação de bocal e pressão de serviço desejada, a forma geométrica mais adequada para representar o perfil radial estacionário adimensional de distribuição de água do aspersor PLONA-RL400. O perfil adimensional selecionado é transformado em sua forma dimensional, pela multiplicação dos valores adimensionais de distância ao aspersor (ra) pelo valor do raio de alcance ( $\mathrm{R}$ em $\mathrm{m}$ ) e pela multiplicação de valores adimensionais de intensidade de precipitação (ia) pelo valor da intensidade média de aplicação de água (im em $\mathrm{mm} \mathrm{h}^{-1}$ ), corrigida em função do ângulo de giro ( $\theta$ em graus), conforme eqs.(1) e (2).

$$
\begin{aligned}
& r_{i}=r a_{i} R \\
& i_{i}=i a_{i} i m \frac{360}{\theta}
\end{aligned}
$$

em que,

$$
\begin{aligned}
& \mathrm{r}_{\mathrm{i}} \text { - distância ao aspersor, } \mathrm{m} \text {; } \\
& \mathrm{i}_{\mathrm{i}} \text { - intensidade de precipitação, } \mathrm{mm} \mathrm{h}^{-1} \text {, e } \\
& \mathrm{i} \text { - índice de identificação de cada coletor. }
\end{aligned}
$$

Em seguida, a lâmina aplicada em diferentes pontos ao longo de um plano perpendicular à trajetória linear de deslocamento do aspersor, operando com diferentes ângulos de giro compreendidos entre 180 e $360^{\circ}$, com intervalos regulares de $30^{\circ}$, é calculada, integrando-se numericamente, em intervalos de tempo correspondentes a 1/40 do tempo total de molhamento em cada ponto, aos valores de intensidades de precipitação experimentados durante o deslocamento do aspersor. Esses valores são sobrepostos, simulando-se a uniformidade de aplicação de água em diferentes espaçamentos entre carreadores, todos múltiplos de $6 \mathrm{~m}$ e compreendidos no intervalo entre 40 e $100 \%$ do diâmetro molhado do aspersor.

Os resultados das simulações de uniformidade de aplicação de água foram agrupados seguindo o mesmo critério estabelecido pelo algoritmo de agrupamento "K-Means". 


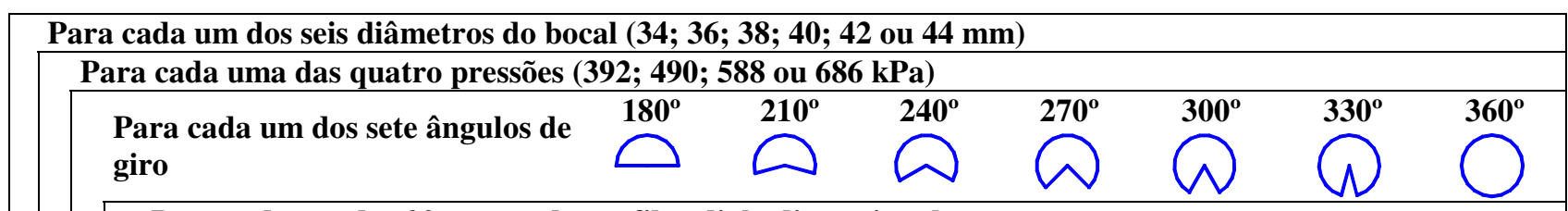

Para cada um dos 20 pontos do perfil radial adimensional

- Dimensionaliza-se a distância ao aspersor [eq.(1)].

- Dimensionaliza-se a intensidade [eq.(2)].

- Apresenta o perfil radial de aplicação de água correspondente à combinação desejada de bocal, pressão de serviço e ângulo de giro, conforme

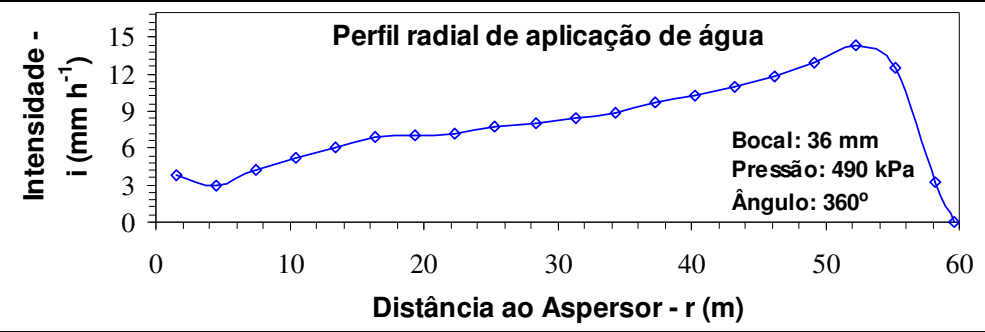
esquematizado ao lado.

\section{Distância ao Aspersor - $r(m)$}

Para cada coletor situado no plano perpendicular ao deslocamento do aspersor

- Determina-se o tempo de molhamento correspondente a uma velocidade de deslocamento do aspersor de $50 \mathrm{~m} \mathrm{~h}^{-1}$.

Para cada instante correspondente a 1/40 do tempo de molhamento

- Determina-se a distância radial entre o coletor e o aspersor e a correspondente intensidade de precipitação de água.

Integração, com auxílio da primeira regra de Simpson, com 40 subintervalos de tempo, das intensidades de precipitação aplicadas no coletor durante o tempo de molhamento

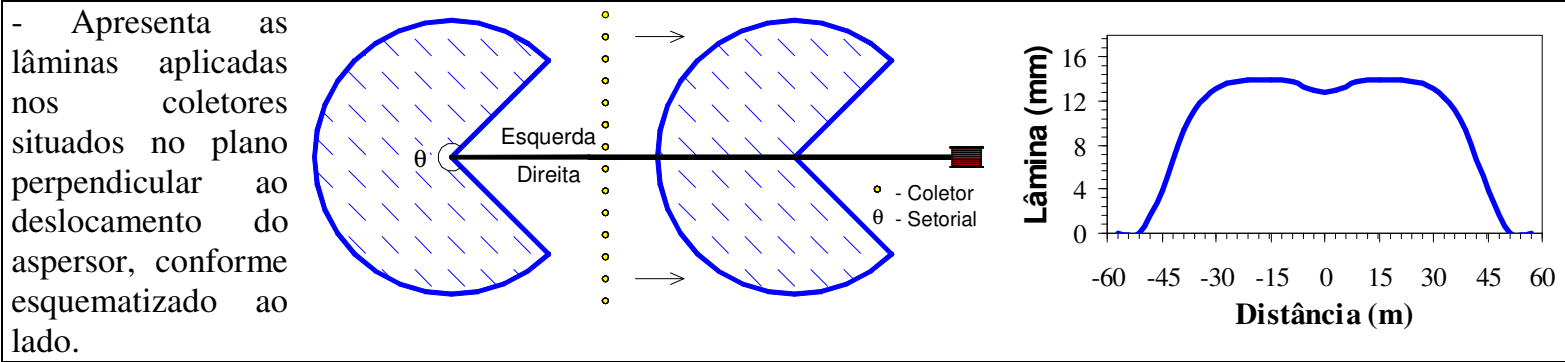

Para espaçamentos múltiplos de 6 m e compreendidos entre 40 a $100 \%$ do diâmetro molhado

Efetua a sobreposição das lâminas aplicadas no plano perpendicular

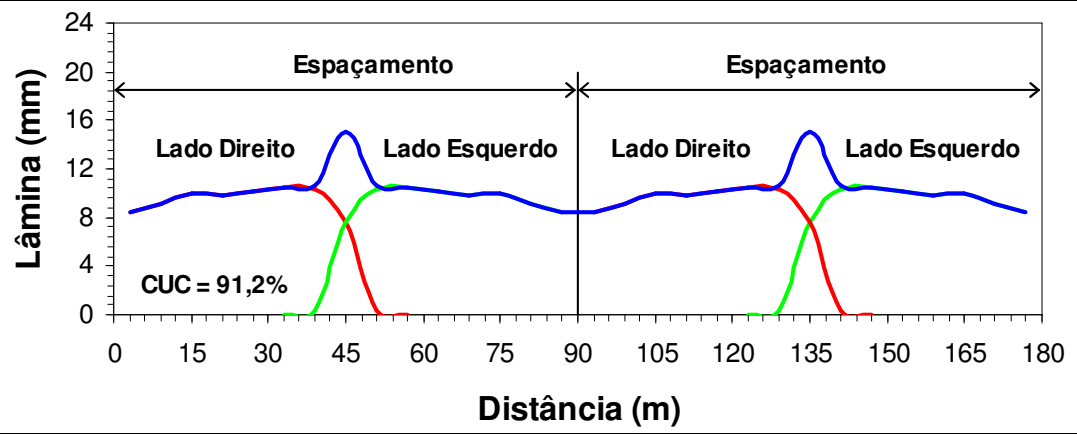

Determina o índice de uniformidade de Christiansen das lâminas sobrepostas.

Imprime o tipo de perfil, o espaçamento entre carreadores em termos da percentagem do diâmetro molhado do aspersor e o índice de uniformidade de Christiansen.

FIGURA 1. Fluxograma do aplicativo computacional desenvolvido em Visual Basic da planilha eletrônica do Excel para a simulação da uniformidade de aplicação de água de sistemas autopropelidos de irrigação.

\section{RESULTADOS E DISCUSSÃO}

Na Figura 2, apresenta-se a variação do erro médio da estimativa da intensidade de precipitação adimensional ( $\left(\mathrm{i}_{\mathrm{i}}\right)$, em função do número de perfis representativos considerados, obtidos na análise de agrupamento com o uso do algoritmo "K-Means". Nota-se que, para três $(\mathrm{K}=3)$ ou mais perfis representativos, há redução gradual menos acentuada no erro médio, justificando-se, portanto, a adoção dos três perfis representativos (I, II e III) ilustrados na Figura 3. 


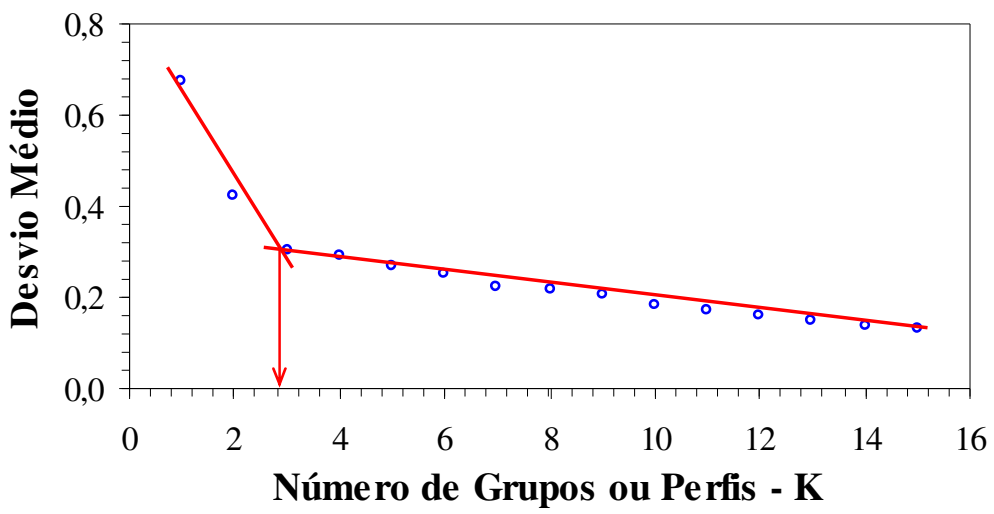

FIGURA 2. Variação do desvio médio da estimativa da intensidade adimensional da aplicação de água em função do número de perfis representativos adotados.
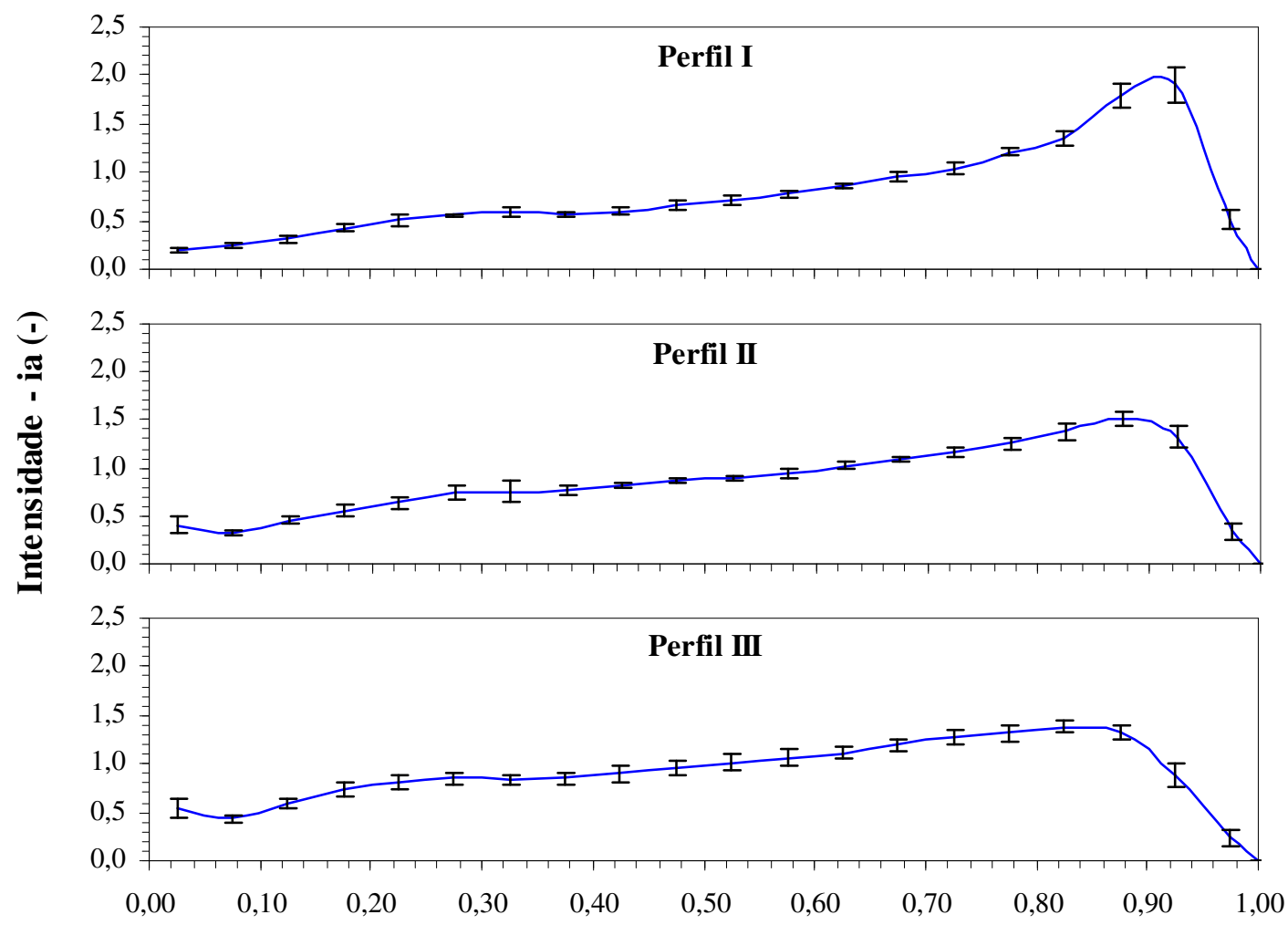

Distância ao aspersor - ra (-)

FIGURA 3. Formas geométricas (I, II e III) assumidas pelo perfil radial adimensional de aplicação de água do aspersor PLONA-RL400, operando de forma estacionária e na ausência de vento, sob diferentes combinações de diâmetro de bocal e pressão de serviço. As barras verticais representam variação de comprimento equivalente a \pm 1 desvio-padrão.

Na Tabela 1, são apresentados, para as 24 diferentes combinações de pressão de serviço e diâmetro do bocal principal consideradas, os valores de vazão, de raio de alcance e do número do perfil adimensional da Figura 3 (I, II ou III) que melhor representa a distribuição radial de água do aspersor PLONA-RL400 ensaiado. Os perfis adimensionais mostrados na Figura 3 (I, II e III) representam condições operacionais do aspersor que determinam três níveis distintos de pulverização do jato de água do aspersor. O perfil I agrupa aquelas condições operacionais que resultam em reduzido grau de pulverização do jato.

A redução no grau de pulverização do Perfil I é inferida por meio da Tabela 1 que, para todos os bocais avaliados, limita a ocorrência do Perfil I à utilização da menor pressão de operação do aspersor. Essa inferência é também mostrada na Figura 3, que apresenta o acúmulo de aplicação de 
água na extremidade do raio de alcance do Perfil I, que é típico de aspersores operando nessa condição (BERNARDO et al., 2005).

O maior grau de pulverização associado à ocorrência do Perfil III é inferido pelos valores da Tabela 1, pela qual se conclui que esse perfil é obtido quando se utiliza a maior pressão de operação do aspersor ou em combinações envolvendo os menores bocais sob elevadas pressões serviço. Essa condição também é confirmada na Figura 3, pela ausência do pico de aplicação na extremidade do raio de alcance do Perfil III.

TABELA 1. Valores observados de vazão, de raio de alcance e do tipo do perfil radial adimensional de aplicação de água do aspersor PLONA-RL400, operando na ausência de vento, com diferentes combinações de diâmetro do bocal principal e pressão de serviço.

\begin{tabular}{|c|c|c|c|c|c|c|c|}
\hline \multirow{2}{*}{$\begin{array}{c}\text { Pressão } \\
\text { kPa } \\
\text { (m.c.a.) }\end{array}$} & \multirow{2}{*}{ Dados Técnicos } & \multicolumn{6}{|c|}{ Diâmetro do Bocal Principal (mm) } \\
\hline & & 34 & 36 & 38 & 40 & 42 & 44 \\
\hline \multirow{3}{*}{$392(40)$} & Vazão $\left(\mathrm{m}^{3} \mathrm{~h}^{-1}\right)$ & 84,45 & 94,01 & 104,29 & 115,38 & 125,34 & 137,05 \\
\hline & Raio (m) & 52,02 & 52,89 & 52,68 & 52,02 & 52,01 & 52,21 \\
\hline & Tipo do Perfil & I & I & $\mathrm{I}$ & $\mathrm{I}$ & $\mathrm{I}$ & $\mathrm{I}$ \\
\hline \multirow{3}{*}{$490(50)$} & Vazão $\left(\mathrm{m}^{3} \mathrm{~h}^{-1}\right)$ & 94,48 & 105,28 & 116,55 & 129,19 & 140,63 & 152,77 \\
\hline & Raio (m) & 59,89 & 59,65 & 60,56 & 60,61 & 60,52 & 59,88 \\
\hline & Tipo do Perfil & II & II & II & II & II & II \\
\hline \multirow{3}{*}{$588(60)$} & Vazão $\left(\mathrm{m}^{3} \mathrm{~h}^{-1}\right)$ & 103,42 & 115,07 & 127,91 & 141,50 & 153,98 & 167,82 \\
\hline & Raio (m) & 64,76 & 65,27 & 65,36 & 65,55 & 66,07 & 66,56 \\
\hline & Perfil & III & III & II & II & II & II \\
\hline \multirow{3}{*}{$686(70)$} & Vazão $\left(\mathrm{m}^{3} \mathrm{~h}^{-1}\right)$ & 111,93 & 124,27 & 138,24 & 152,96 & 165,37 & 181,23 \\
\hline & Raio (m) & 69,36 & 68,62 & 69,39 & 70,34 & 70,49 & 71,43 \\
\hline & Tipo do Perfil & III & III & III & III & III & III \\
\hline
\end{tabular}

Condições operacionais que conduzem ao grau intermediário de pulverização do jato são agrupadas e representadas pelo Perfil II. A transição gradual do formato geométrico assumido pelo perfil radial de aplicação de água do aspersor, em função da mudança gradual das condições operacionais, que é mostrada na Tabela 1, demonstra que a adimensionalização proposta por SOLOMON \& BEZDEK (1980) permite, também, avaliar, em função da tendência geral observada, a adequação de cada resultado individual de ensaio obtido.

O poder de síntese da análise de agrupamento é também demonstrado na Figura 4, que permite selecionar, para as diferentes condições operacionais mostradas na Tabela 1, as combinações de espaçamento entre carreadores e ângulo de giro do aspersor que resultam em valores mais elevados de uniformidade de aplicação (uniformidade de Christiansen - CUC), na parte central da faixa irrigada por equipamento autopropelido operando com o aspersor PLONARL400. Observa-se que, para os perfis I, II e III (Figura 4a, 4b e 4c), na ausência de ventos, independentemente do ângulo de giro, os maiores valores de uniformidade são obtidos para valores de espaçamento entre carreadores menores que $50 \%$ do diâmetro molhado do aspersor e para valores entre 80 e $90 \%$ do diâmetro molhado do aspersor.

A recomendação de espaçamento entre carreadores, inferida pelos valores mostrados na Figura 4, excede o valor de $80 \%$ do diâmetro molhado sugerido na literatura (KELLER \& BLIESNER, 1990; TARJUELO, 1999 e BERNARDO et al., 2005) para a condição de ventos leves. No que diz respeito ao ângulo de giro do aspersor na faixa de espaçamentos recomendados, para os três perfis, os melhores valores de CUC são observados (Figura 4) quando o ângulo de giro se situa entre 330 e $360^{\circ}$ ou entre 180 e $230^{\circ}$, confirmando o intervalo de ângulos de giro do aspersor de 200 a $220^{\circ}$ recomendado por KELLER \& BLIESNER (1990) e TARJUELO (1999). 


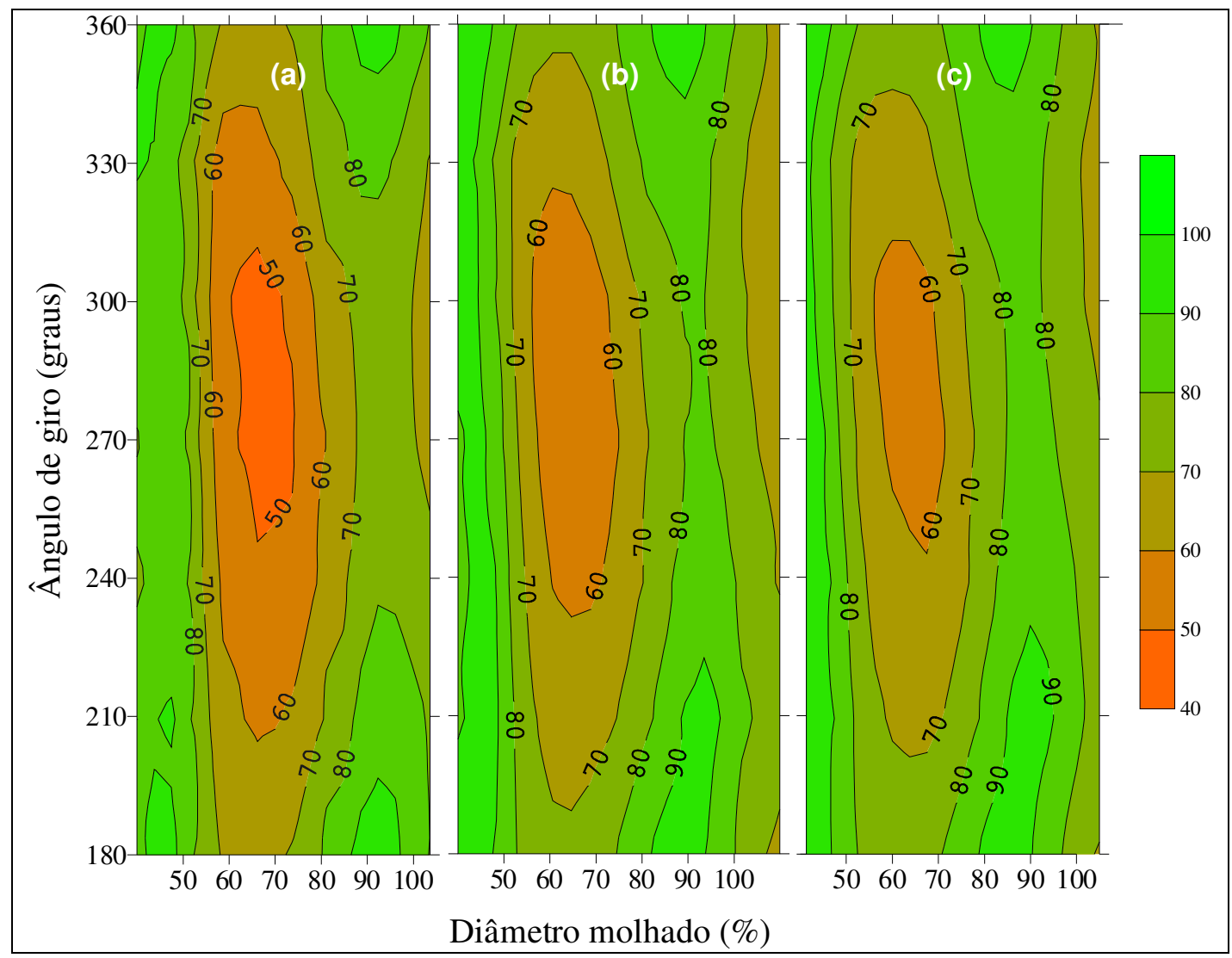

FIGURA 4. Coeficiente de Uniformidade de Christiansen (CUC) em função do ângulo de giro e do espaçamento entre carreadores para as formas geométricas I (a), II (b) e III (c), assumidas do perfil radial adimensional de aplicação de água do aspersor PLONARL400.

Observa-se, na Figura 4, que a utilização de espaçamentos entre 60 e $70 \%$ do diâmetro molhado, a qual é recomendada por BERNARDO et al. (2005), para ventos na faixa de 2,2 a $4,7 \mathrm{~m} \mathrm{~s}^{-1}$, não assegura a obtenção de valores adequados de uniformidade na condição de ausência de ventos. Considerando-se as limitações nos valores de espaçamento entre carreadores impostas pelos valores de velocidade de vento que são descritas na literatura, e os resultados de uniformidade de aplicação de água mostrados na Figura 4, para a condição de ausência de vento, pode-se inferir que espaçamentos entre carreadores menores que 50\% são adequados para ambas as condições de vento consideradas.

\section{CONCLUSÕES}

Com base nos resultados obtidos, conclui-se que, para o aspersor PLONA-RL400, operando em condições de ausência de ventos: $i$ ) três formas geométricas adimensionais podem ser utilizadas para representar os perfis radiais de distribuição de água do aspersor; ii) espaçamentos de carreadores menores que 50\% do diâmetro molhado e compreendidos entre 80 e $90 \%$ do diâmetro molhado do aspersor resultam em adequados valores de CUC, e iii) ângulos de giro entre $180 \mathrm{e}$ $210^{\circ}$ geram melhores valores de uniformidade de aplicação de água.

\section{AGRADECIMENTOS}

À empresa PLONA Equipamentos (Curitiba - PR), por disponibilizar o aspersor RL400 para a realização dos ensaios técnicos.

\section{REFERENCIAS}

BERNARDO, S.; SOARES, A.A.; MANTOVANI ,.E.C. Manual de Irrigação. 7.ed. Viçosa: Imprensa Universitária, 2005. 611 p. 
BITTINGER, M. W.; LONGENBAUGH, R. A. Theoretical distribution of water from a moving irrigation sprinkler. Transactions of the ASAE, St. Joseph, v.5, n.1, p. 26-30, jan./feb. 1962.

KELLER, J.; BLIESNER, R.D. Sprinkler and trickle irrigation. New York: Van Nostrand Reinhold, 1990. 652 p.

PRADO, G. Aplicativo computacional para simulação da distribuição de água pelo aspersor PLONA-RL300 em sistemas autopropelidos de irrigação. 2004. 86 f. Dissertação (Mestrado em Irrigação e Drenagem) - Universidade Federal de Lavras, Lavras - MG, 2004.

PRADO, G.; COLOMBO, A. Caracterização técnica do aspersor PLONA-RL300. Irriga, Botucatu, v.10, n.1, p.53-63, 2005.

ROCHA, F.A.; PEREIRA, G.M.; ROCHA, F.S.; SILVA, J.O. Análise da uniformidade de distribuição de água de um equipamento autopropelido. Irriga, Botucatu, v.10, n.1, p.96-106, 2005.

ROLIM, J.; PEREIRA, L.S. Design and evaluation of traveling-gun systems: the simulation model TRAVGUN. In: EFITA/WCCA JOINT CONGRESS ON IT IN AGRICULTURE, 2005, Vila Real. Proceedings... Vila Real: EFITA/WCCA, 2005. p.166-71.

SOLOMON, K.; BEZDEK, J.C. Characterizing sprinkler distribution patterns with a clustering algorithm. Transactions of the ASAE, St. Joseph, v.23, n.4, p.899-906, july/aug. 1980.

TARJUELO, J. M. El riego por aspersión y su tecnología. $2^{\text {nd }}$.ed. Madrid: Mundi Prensa, 1999. $529 \mathrm{p}$.

TOU, J.T.; GONZALES, R.C. Pattern recognition principles. London: Addison-Wesley, 1974. $377 \mathrm{p}$. 